\title{
The survival strength of younger patients in BCLC stage $0-B$ of hepatocellular carcinoma: basing on competing risk model
}

Huiwen Yan ${ }^{1,2+}$, Xinhui Wang ${ }^{1 \dagger}$, Xiaoli Liu ${ }^{1}$, Peng Wang ${ }^{1}$, Lihua Yu ${ }^{1}$, Dongdong Zhou ${ }^{1}$ and Zhiyun Yang ${ }^{1 *}$

\begin{abstract}
Background: The number of young patients with hepatocellular carcinoma $(\mathrm{HCC})$ is increasing, but whether patients of different ages have a survival advantage is unclear. This study was conducted to investigate whether age differences in the Barcelona Clinic Liver Cancer (BCLC) classification system contribute to the long-term survival outcomes of patients with HCC.

Methods: A total of 1602 patients with HCC admitted to the Beijing Ditan Hospital was included in this study. Patients were divided into younger ( $\leq 45$ years) and older ( $>45$ years) groups. Factors determining overall survival and progression-free survival were analyzed using univariate and multivariate analyses with the Kaplan-Meier method and Cox proportional hazard regression model. We calculated the cumulative incidence function using the Fine-Gray model. The effect of mortality on age was also estimated using a restricted cubic spline.
\end{abstract}

Results: After matching, overall survival and progression-free survival were significantly better in younger patients than in older patients with BCLC stage $0-B(p=0.015$ and $p=0.017$, respectively). In BCLC stage $0-B$, all-cause mortality increased with age and increased rapidly around the age of 40 years (non-linear, $p<0.05$ ). In BCLC stages 0-B, HCCrelated and non-HCC-related deaths significantly differed between younger and older individuals $(p=0.0019)$.

Conclusion: In stage BCLC 0-B, age affects the long-term prognosis of patients.

Keywords: Hepatocellular carcinoma, Younger, Barcelona clinic liver Cancer classification, Prognosis, Survival

\section{Introduction}

Hepatocellular carcinoma (HCC), a malignant lesion of the liver cells, is a major diseases that seriously endangers human life and health, and is a major public health issue. More than 500,000 new cases of HCC are diagnosed globally each year $[1,2]$. Hepatitis B virus (HBV) and hepatitis $\mathrm{C}$ virus $(\mathrm{HCV})$ infection are the two most important causes of $\mathrm{HCC}$, with $\mathrm{HBV}$ associated with

\footnotetext{
*Correspondence: yangzhiyun2016@163.com

${ }^{\dagger}$ Huiwen Yan and Xinhui Wang contributed equally to this work.

${ }^{1}$ Center of Integrative Medicine, Capital Medical University Affiliated Beijing Ditan Hospital, No. 8 Jing Shun East Street, Beijing 100015, People's Republic of China

Full list of author information is available at the end of the article
}

HCC in $60-70 \%$ of patients in Asian countries, and $\mathrm{HCV}$ infection in $60-80 \%$ of patients in western countries. Liver cancer mainly occurs in older populations ( $>45$ years of age) with a higher incidence of comorbidities, which are typically considered as high-risk groups for treatment [3]. The incidence of liver cancer in younger patients is increasing with improvements in liver cancer screening among high-risk groups $[4,5]$. Several studies showed that the prognosis of elderly patients with HCC is worse than that of younger patients, whereas other studies reported a worse prognosis in younger patients or no difference in prognosis between younger and older patients [6-9]. 
The Barcelona Clinical Liver Cancer (BCLC) staging system provides better predictive value for early diagnosis. Most patients are initially diagnosed with mid- to late-stage liver cancer in a vague manner $[10,11]$. As a result, the BCLC staging system has been widely used for the diagnosis and evaluation of HCC.

These contradictory results may be related to comparisons between different age groups, regardless of the tumor staging. We compared the survival outcomes of HCC in older ( $>45$ years) and younger ( $\leq 45$ years) patients according to BCLC staging.

\section{Materials and methods}

\section{Diagnosis and staging of HCC}

We conducted a retrospective cohort study of 1602 patients with HCC using data from the Beijing Ditan Hospital from January 2009 to December 2018. HCC was diagnosed based on the recommendations of the European Association for the Study of Liver Diseases and the American Association for the Study of Liver Diseases (AASLD). The inclusion criteria were as follows: (1) clear clinical diagnosis of HCC and (2) patients with complete clinical data. Exclusion criteria included: (1) autoimmune liver disease; hepatitis A, D, or E; syphilis; acquired immunodeficiency syndrome; or other primary malignancy; (2) metastatic liver cancer; (3) pregnant women; and (4) patients with unclear BCLC staging. The primary outcome was the death of patients with HCC, with the observation time defined as the time between the patient's first participation in this observational study and date of death. Patient survival was defined as the time between the diagnosis of liver cancer and death or the end of the study.

\section{Definition of BCLC stages}

The BCLC staging system contains four main categories of prognostic factors: the patient's general state, tumor state, liver function state, and available treatment options (2018 AASLD) [12]. The very early stage (0) is defined as solitary $\mathrm{HCC}(<2 \mathrm{~cm})$, early stage (A) is solitary or $2-3$ nodules $(\leq 3 \mathrm{~cm})$, intermediate stage $(B)$ is multinodular and unresectable, advanced stage $(\mathrm{C})$ is the presence of portal invasion or extrahepatic spread, and terminal stage (D) is a severe physical condition (Performance Status (PS) score of 3-4) and end-stage liver function. We compared the demographic data and clinical factors between the two groups (BCLC stage 0-B and BCLC stage C-D).

\section{Demographics and clinical data}

We extracted the following data for the study: sex, age, family history of HCC, alcohol abuse, esophageal and/or gastric varices, $\mathrm{HBsAg}, \mathrm{HCV}$, cirrhosis, portal vein tumor thrombus (PVTT), Child-Pugh score, tumor multiplicity, tumor size, white blood cell (WBC), platelet counts (PLT), alanine aminotransferase (ALT) and treatment at baseline. Laboratory values at the time of diagnosis of $\mathrm{HCC}$ are classified as elevated when they are higher than the clinical normal values. Tumor staging was performed using the BCLC staging method and liver function was assessed using the Child-Pugh scoring method.

\section{Statistical analysis}

Demographics and clinical factors were compared between the two groups (age $>45$ and $\leq 45$ years groups), with categorical variables using the chi-square test. Overall survival (OS) and progression-free survival (PFS) were estimated using the Kaplan-Meier method and compared using the log-rank test. OS was defined as the period from the first diagnosis to death, independent of the date of the last follow-up. PFS was defined as the time between the first diagnosis and date of diagnosis of tumor progression or last follow-up. Cox proportional regression was used to determine age factors independently associated with BCLC stage $0-\mathrm{B}$. To assess prognostic factors independent of age, variables identified as significant in univariate analysis were included in the multivariate Cox proportional hazards model.

We further assessed the pattern of association between age and primary outcome using a Cox proportional hazards regression model with restricted cubic splines (RCS) for age, with 6 knots at 30, 40, 50, 60, 70, and 80 years of age. To determine the effect of age on HCC-related and non-HCC-related causes of death, we calculated a cumulative incidence function using the Fine-Gray model. HCC-related deaths included death from HCC and/or rupture of intra-abdominal liver cancer. Non-HCC death occurred because of a condition other than those mentioned above.

Statistical significance was set at $p<0.05$. All statistical analyses were performed using $\mathrm{R}$ version 3.6.3 (The $\mathrm{R}$ Foundation for Statistical Computing, Vienna, Austria).

\section{Results \\ Clinical characteristics of the study participants}

Table 1 shows the demographic and clinical characteristics of the two groups based on age. The cohort included 1395 patients with HCC aged > 45 years and 207 patients with $\mathrm{HCC}$ aged $\leq 45$ years. Prior to PS matching, the younger group of patients contained more men and showed a lower proportion of cirrhosis compared to the older patient group (Table 1$)(p<0.001)$. In addition, younger patients had a higher proportion of WBC, PLT and ALT. Because of the heterogeneity between younger and older patients at baseline, PS matching was performed for sex, BCLC staging, and three treatment types. Thereafter, younger and older patients were essentially 
Table 1 Demographic and clinical characteristics of study patients

\begin{tabular}{|c|c|c|c|c|}
\hline Demographic and clinical values & Total $(N=1602)$ & Age group $>45 y(N=1395)$ & Age group $\leq 45 y(N=207)$ & $P$ value \\
\hline \multicolumn{5}{|l|}{ Patient characteristics } \\
\hline Sex & & & & $<0.001$ \\
\hline Male & $1254(78.3)$ & $1071(76.8)$ & $183(88.4)$ & \\
\hline Female & $348(21.7)$ & $324(23.2)$ & $24(11.6)$ & \\
\hline Alcohol abuse & & & & 0.812 \\
\hline No alcohol & $991(61.9)$ & $865(62.0)$ & $126(60.9)$ & \\
\hline Alcohol & $611(38.1)$ & $530(38.0)$ & $81(39.1)$ & \\
\hline Family history of HCC & & & & 0.207 \\
\hline No & $1545(96.4)$ & $1349(96.7)$ & $196(94.7)$ & \\
\hline Yes & $57(3.6)$ & $46(3.3)$ & $11(5.3)$ & \\
\hline HBsAg & & & & 0.001 \\
\hline Negative & $172(10.7)$ & $164(11.8)$ & $8(3.9)$ & \\
\hline Positive & $1430(89.3)$ & $1231(88.2)$ & $199(96.1)$ & \\
\hline $\mathrm{HCV}$ & & & & 0.006 \\
\hline Negative & $1532(95.6)$ & $1326(95.1)$ & $206(99.5)$ & \\
\hline Positive & $70(4.4)$ & $69(4.9)$ & $1(0.5)$ & \\
\hline Esophageal and/or gastric varices & & & & 0.667 \\
\hline No & $1231(76.8)$ & $1069(76.6)$ & $162(78.3)$ & \\
\hline Yes & $371(23.2)$ & $326(23.4)$ & $45(21.7)$ & \\
\hline Cirrhosis & & & & $<0.001$ \\
\hline No & $113(7.1)$ & $80(5.7)$ & $33(15.9)$ & \\
\hline Yes & $1489(92.9)$ & $1315(94.3)$ & $174(84.1)$ & \\
\hline PVTT at baseline & & & & 0.031 \\
\hline No & $1264(78.9)$ & $1113(79.8)$ & $151(72.9)$ & \\
\hline Yes & $338(21.1)$ & $282(20.2)$ & $56(27.1)$ & \\
\hline Child-Pugh Staging & & & & 0.003 \\
\hline A & $784(48.9)$ & $660(47.3)$ & $124(59.9)$ & \\
\hline B & $589(36.8)$ & $531(38.1)$ & $58(28.0)$ & \\
\hline C & $229(14.3)$ & $204(14.6)$ & $25(12.1)$ & \\
\hline \multicolumn{5}{|l|}{ Tumor characteristics } \\
\hline Tumor multiplicity & & & & 0.306 \\
\hline Solitary & $903(56.4)$ & $779(55.8)$ & $124(59.9)$ & \\
\hline Multiple & $699(43.6)$ & $616(44.2)$ & $83(40.1)$ & \\
\hline Tumor size & & & & 0.186 \\
\hline$\leq 5 \mathrm{~cm}$ & $1097(68.5)$ & $964(69.1)$ & $133(64.3)$ & \\
\hline$>5 \mathrm{~cm}$ & $505(31.5)$ & $431(30.9)$ & $74(35.7)$ & \\
\hline BCLC staging & & & & 0.257 \\
\hline $0-B$ & $1058(66.0)$ & $929(66.6)$ & $129(62.3)$ & \\
\hline$C-D$ & $544(34.0)$ & $466(33.4)$ & $78(37.7)$ & \\
\hline \multicolumn{5}{|l|}{ Preoperative laboratory tests } \\
\hline WBC (10^9/L) & & & & $<0.001$ \\
\hline Low $\leq 4$ & $673(42.0)$ & $614(44.0)$ & $59(28.5)$ & \\
\hline High>4 & 929 (58.0) & 781 (56.0) & $148(71.5)$ & \\
\hline $\operatorname{PLT}(10 \wedge 9 / L)$ & & & & $<0.001$ \\
\hline Low $\leq 100$ & $903(56.4)$ & $814(58.4)$ & $89(43.0)$ & \\
\hline High> 100 & $699(43.6)$ & $581(41.6)$ & $118(57.0)$ & \\
\hline ALT (U/L) & & & & 0.002 \\
\hline Low $\leq 50$ & $1166(72.8)$ & $1034(74.1)$ & $132(63.8)$ & \\
\hline High> 50 & $436(27.2)$ & $361(25.9)$ & 75 (36.2) & \\
\hline
\end{tabular}


Table 1 (continued)

\begin{tabular}{llll}
\hline Demographic and clinical values & Total $(\boldsymbol{N}=\mathbf{1 6 0 2})$ & Age group $>\mathbf{4 5 y}(\boldsymbol{N}=\mathbf{1 3 9 5})$ & Age group $\leq \mathbf{4 5 y}(\boldsymbol{N}=\mathbf{2 0 7}) \quad \boldsymbol{P \text { value }}$ \\
\hline $\begin{array}{l}\text { Type of treatment } \\
\text { Resection }\end{array}$ & & \\
$\quad$ No & $1547(96.6)$ & $1352(96.9)$ & $195(94.2)$ \\
$\quad$ Yes & $55(3.4)$ & $43(3.1)$ & $12(5.8)$ \\
Palliative & $1199(74.8)$ & $1044(74.8)$ & $155(74.9)$ \\
$\quad$ No & $403(25.2)$ & $351(25.2)$ & $52(25.1)$ \\
$\quad$ Yes & & & 7.072 \\
Minimally invasive & $536(33.5)$ & $460(33.0)$ & $76(36.7)$ \\
$\quad$ No & $1066(66.5)$ & $935(67.0)$ & $131(63.3)$ \\
$\quad$ Yes & & 0.324 \\
\hline
\end{tabular}

$p$ value between $>45 y$ and $\leq 45 y$ age groups

PVTT portal vein tumor thrombus, WBC white blood cell, PLT platelet, $A L T$ alanine aminotransferase

identical in terms of tumor characteristics and type of treatment (Table S1).

\section{Survival analysis}

Age was an independent predictor of survival in BCLC stages 0-B before (Fig. 1A-D) and after PS matching (Fig. 1E-H). Young patients showed better 5-year OS and PFS compared to older patients $(p=0.015$ and $p=0.017$, respectively; Fig. 1E, F). However, in BCLC C-D, there were no significant differences in OS and PFS between younger and older patients $(p=0.66$ and $p=0.97$, respectively; Fig. 1G, H).

\section{RCS of patient death versus age for different BCLC stages}

We also used RCS to model the association between age and mortality at $40,50,60,70$, and 80 years of age. The hazard ratio (HR) at 45 years of age was defined as 1 based on the patient's age HR and its $95 \%$ confidence interval $(\mathrm{CI})$, and was divided into BCLC stages $0-\mathrm{B}$ and $\mathrm{C}-\mathrm{D}$, as shown in Fig. 2. In BCLC stage 0-B, all-cause
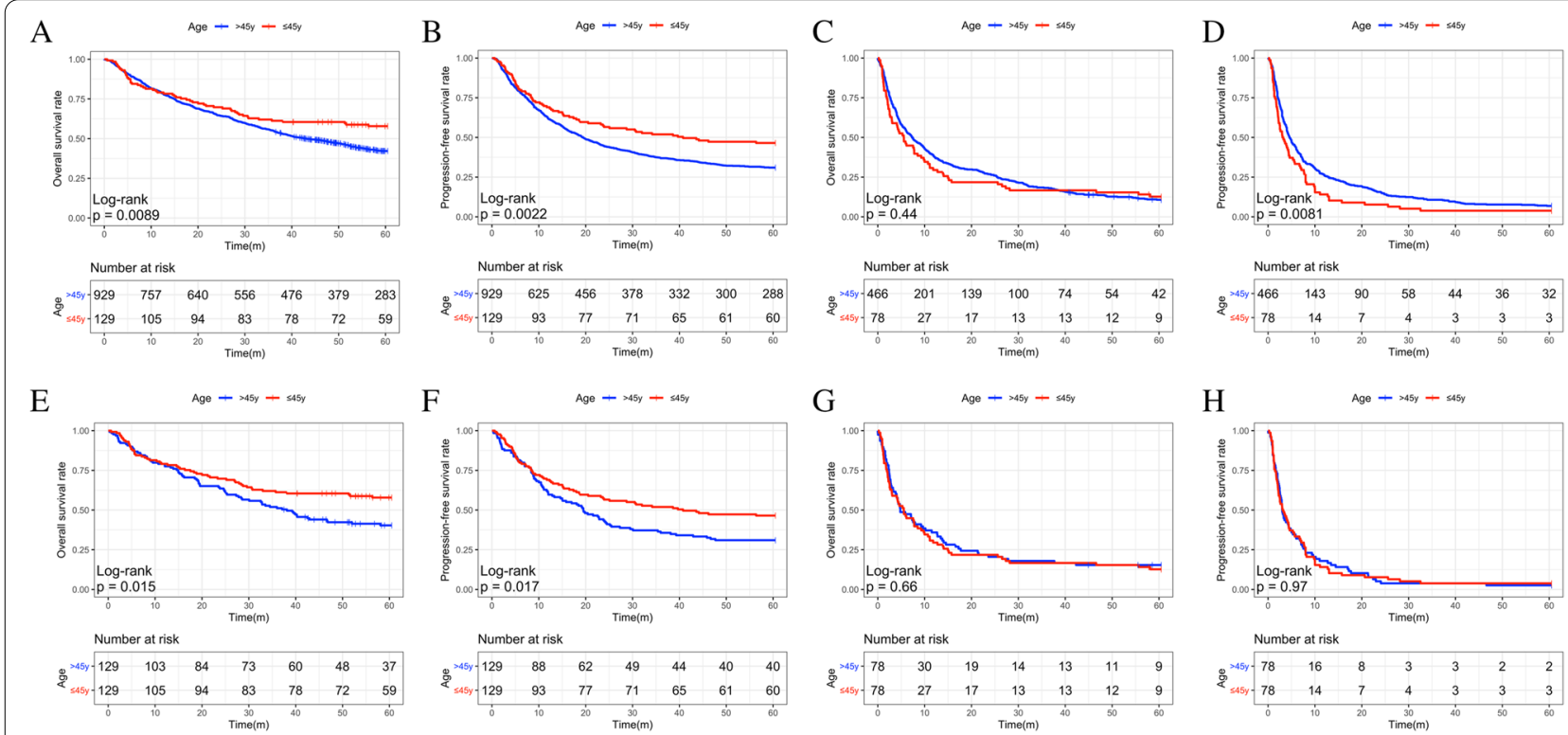

G

$\mathrm{H}$
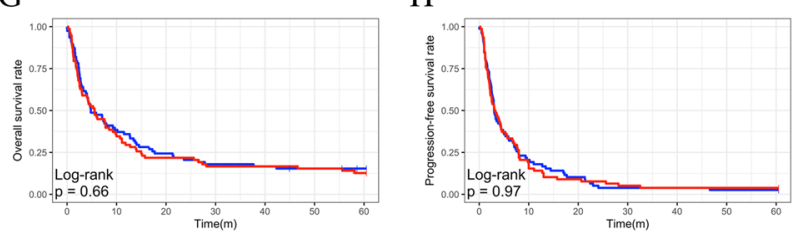

Number at risk
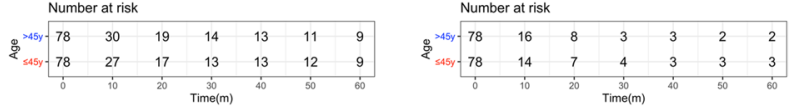

Fig. 1 The Kaplan-Meier survival curves of overall survival (OS) and progression-free survival (PFS) in different BCLC stage HCC patients before and after PSM. A,B The OS (A) and PFS (B) in BCLC stage 0-B before PSM. C,D The OS (C) and PFS (D) in BCLC stage C-D before PSM. E,F The OS (E) and PFS (F) in BCLC stage 0-B after PSM. G,H The OS (H) and PFS (H) in BCLC stage C-D after PSM. HCC, hepatocellular carcinoma; BCLC, Barcelona Clinic Liver Cancer; PSM, Propensity Score Matching 


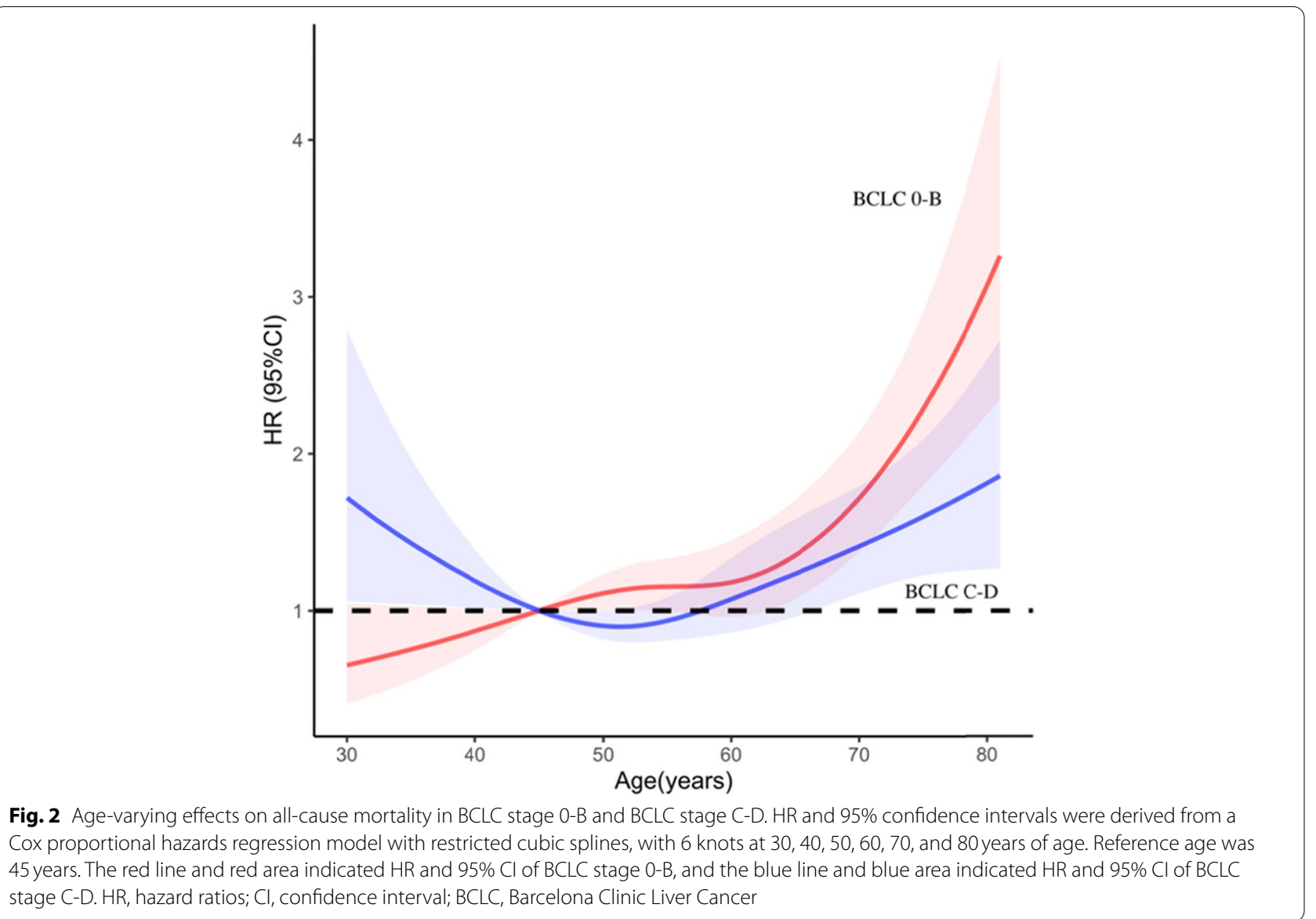

mortality increased with age, with a rapid increase at around age 60 years (nonlinear, $p<0.05$ ). There was a significant $\mathrm{U}$-shaped relationship between age and allcause mortality in BCLC stage C-D; there was a significant reduction in risk at the lower age range, reaching a minimum risk around age 51 years followed by an increase (nonlinear $\mathrm{p}<0.05$ ).

\section{Fine-gray model of the two causes of death for different BCLC stages}

The 5-year outcomes of the two causes of death, HCCrelated death and non-HCC-related death, significantly differed for BCLC stage 0 -B patients aged $\leq 45$ years and older $(p=0.0019)$; non-HCC-related death was not significantly different $(p=0.2897)$. The cumulative incidence functions at month 50 were 52.9, 35.0, 4.9 , and $7.1 \%$, respectively, for patients aged $\leq 45$ years and older BCLC stage C-D $(p=0.3581, p=0.5462$, respectively) and the cumulative incidence functions at month 50 was $86.9,86.0,2.4$, and $1.3 \%$, respectively (Fig. 3A and B).

\section{Univariate and multivariate analyses of BCLC stages 0-B}

A multivariate Cox proportional risk model was used to analyze the influence of baseline indicators on the 5 -year mortality of patients with liver cancer (Table 2). Being younger $(\mathrm{HR}=0.74,95 \% \mathrm{CI}, 0.55-0.98, p=0.037)$ and having a tumor size $\geq 5 \mathrm{~cm}(\mathrm{HR}=2.46,95 \% \mathrm{CI}$, $2.05-2.95, p<0.001)$ were found to be independent risk factors affecting OS. The independent protective factors of PFS in patients with HCC (Table 2) were younger age $(\mathrm{HR}=0.71,95 \% \mathrm{CI}, 0.55-0.91, p=0.008)$ and tumor size $\geq 5 \mathrm{~cm}(\mathrm{HR}=2.41,95 \% \mathrm{CI}, 2.04-2.85, \mathrm{p}<0.001)$. A younger age showed the strongest protective effect after multivariate adjustment.

\section{Subgroup analysis of BCLC stages 0-B}

Subgroup analysis of OS revealed a $26.1 \%$ reduction in the risk of death in young men $(\mathrm{HR}=0.739 ; 95 \% \mathrm{CI}$, 0.552-0.988) (Fig. 4A). Compared with older patients, the risk of death was $35.4 \%$ lower in young men without esophageal or gastric varices $(\mathrm{HR}=0.646 ; 95 \%$ $\mathrm{CI}, 0.47-0.888)$ and $41.4 \%$ lower in young men with tumor size $\leq 5 \mathrm{~cm}(\mathrm{HR}=0.586 ; 95 \% \mathrm{CI}, 0.412-0.833)$. 

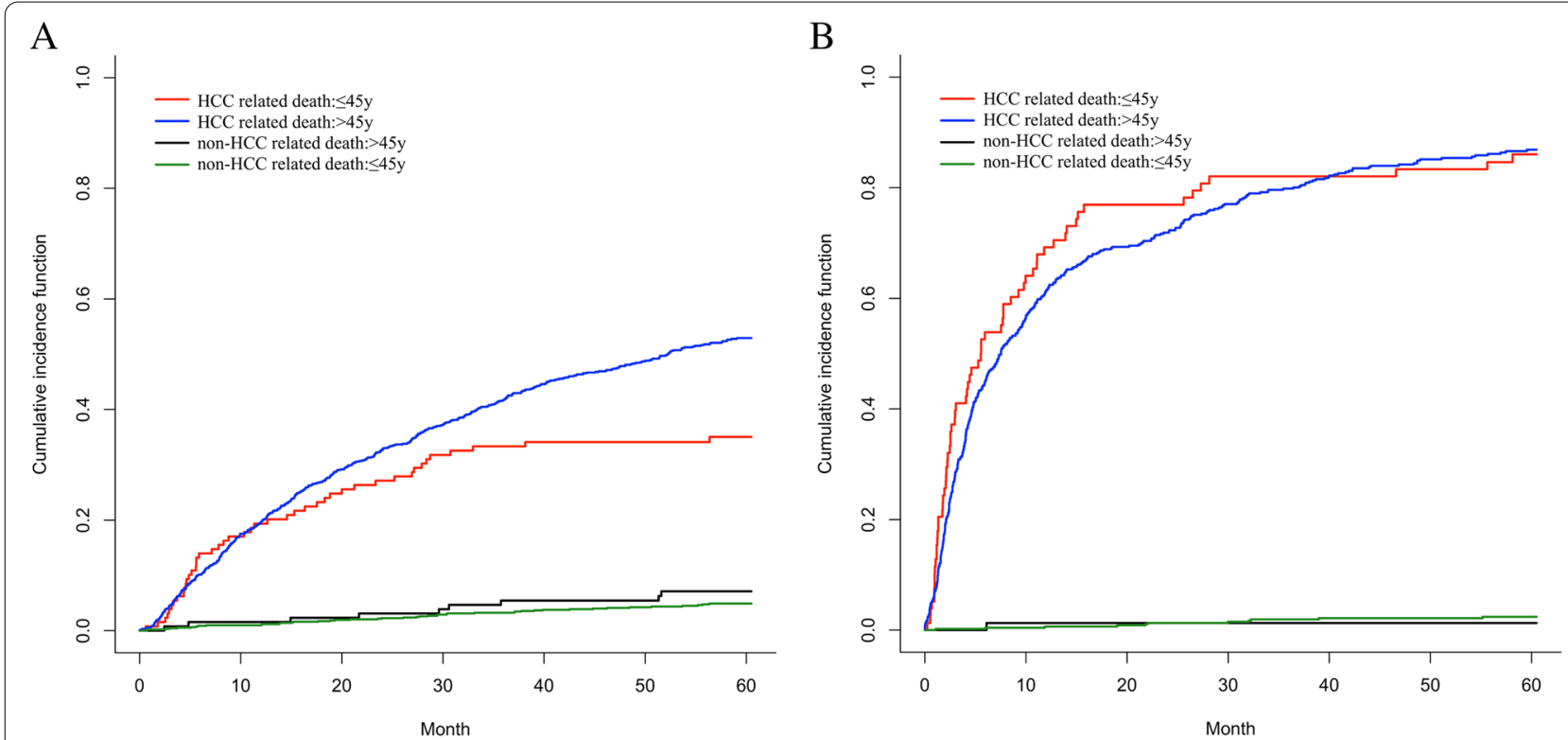

Fig. 3 In BCLC 0-B group (A) and BCLC C-D group (B), overall survival by cause of death was observed in $>45 y$ and $\leq 45 y$ age groups. The curves were estimated based on a Fine-Gray model. HCC, hepatocellular carcinoma

Table 2 Prognostic factor analysis for overall survival and progression-free survival

\begin{tabular}{|c|c|c|c|c|c|c|c|c|c|c|c|c|}
\hline \multirow[t]{3}{*}{ Variables } & \multicolumn{6}{|c|}{ Overall survival } & \multicolumn{6}{|c|}{ Progression-free survival } \\
\hline & \multicolumn{3}{|c|}{ Univariate analysis } & \multicolumn{3}{|c|}{ Multivariate analysis } & \multicolumn{3}{|c|}{ Univariate analysis } & \multicolumn{3}{|c|}{ Multivariate analysis } \\
\hline & HR & $95 \% \mathrm{Cl}$ & p.value & HR & $95 \% \mathrm{Cl}$ & p.value & HR & $95 \% \mathrm{Cl}$ & p.value & HR & $95 \% \mathrm{Cl}$ & p.value \\
\hline Sex[Female] & 0.93 & $0.76-1.1$ & 0.45 & & & & 0.93 & $0.78-1.1$ & 0.44 & & & \\
\hline Age group $[\leq 45 y]$ & 0.69 & $0.52-0.91$ & 0.0093 & 0.74 & $0.55-0.98$ & 0.037 & 0.68 & $0.53-0.87$ & 0.0023 & 0.71 & $0.55-0.91$ & 0.008 \\
\hline Alcohol abuse[alcohol] & 1.2 & $0.98-1.4$ & 0.079 & & & & 1.1 & $0.95-1.3$ & 0.21 & & & \\
\hline Family history of HCC[No] & 0.75 & $0.45-1.3$ & 0.28 & & & & 0.91 & $0.59-1.4$ & 0.65 & & & \\
\hline Tumor size $[>5 \mathrm{~cm}]$ & 2.4 & $2-2.9$ & $<0.001$ & 2.46 & $2.05-2.95$ & $<0.001$ & 2.4 & $2-2.8$ & $<0.001$ & 2.41 & $2.04-2.85$ & $<0.001$ \\
\hline PVTT at baseline[Yes] & 2 & $0.82-4.8$ & 0.13 & & & & 1.3 & $0.55-3.2$ & 0.53 & & & \\
\hline WBC (10^9/L) [High>4] & 0.97 & $0.83-1.1$ & 0.75 & & & & 0.95 & $0.82-1.1$ & 0.46 & & & \\
\hline PLT(10^9/L) [High> 100] & 0.95 & $0.81-1.1$ & 0.56 & & & & 0.99 & $0.85-1.1$ & 0.89 & & & \\
\hline ALT(U/L) $[H i g h>50]$ & 1.2 & $0.96-1.4$ & 0.12 & & & & 1.2 & $1-1.4$ & 0.035 & & & \\
\hline
\end{tabular}

A Cox proportional hazards regression model for OS and PFS was used. The reference category for each categorical variable is in the square brackets in first column. HR, hazard ratio; OS, overall survival; PFS, progression-free survival; PVTT, portal vein tumor thrombus; WBC, white blood cell; PLT, platelet; ALT, alanine aminotransferase

Subgroup analysis of PFS showed that compared with older patients, young people without esophageal or gastric varices had a $31.6 \%$ lower risk of death compared with patients ( $\mathrm{HR}=0.684 ; 95 \% \mathrm{CI}, 0.521-0.898)$ (Fig. 4B). Young people with a tumor size $\leq 5 \mathrm{~cm}$ had a $38.4 \%$ lower risk of death $(\mathrm{HR}=0.616$; 95\% CI, 0.456-0.832). Patients with BCLC stage 0-B tumor size $\leq 5 \mathrm{~cm}$ in size had better 5 -year OS and PFS compared with patients with tumor size $>5 \mathrm{~cm}$ (both $p<0.0001$; Fig. S1A, B). In contrast, patients with esophageal or gastric varices had a better 5-year OS compared to those without varices
( $p=0.0019)$, whereas the PFS was not significantly different between these groups ( $p=0.2$; Fig. S1C, D).

\section{Discussion}

Patient age is an important prognostic factor in various malignancies. In some cancers, the prognosis is poorer in young people, whereas cancers show good survival outcomes in middle-aged patients [13]. Whether age affects the survival of patients with liver cancer is unclear. According to American guidelines for the management of $\mathrm{HCC}$, screening should begin at the age of 40 years 

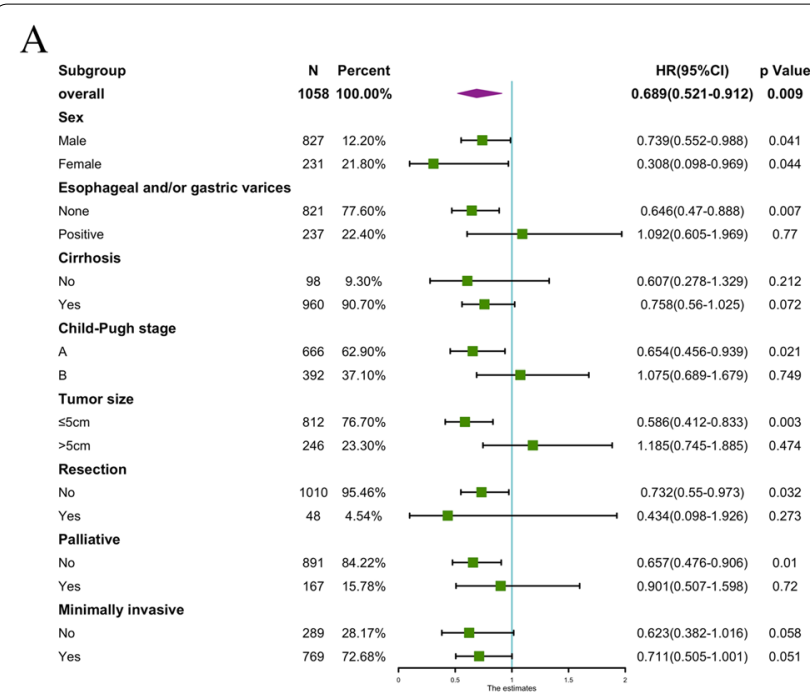

B

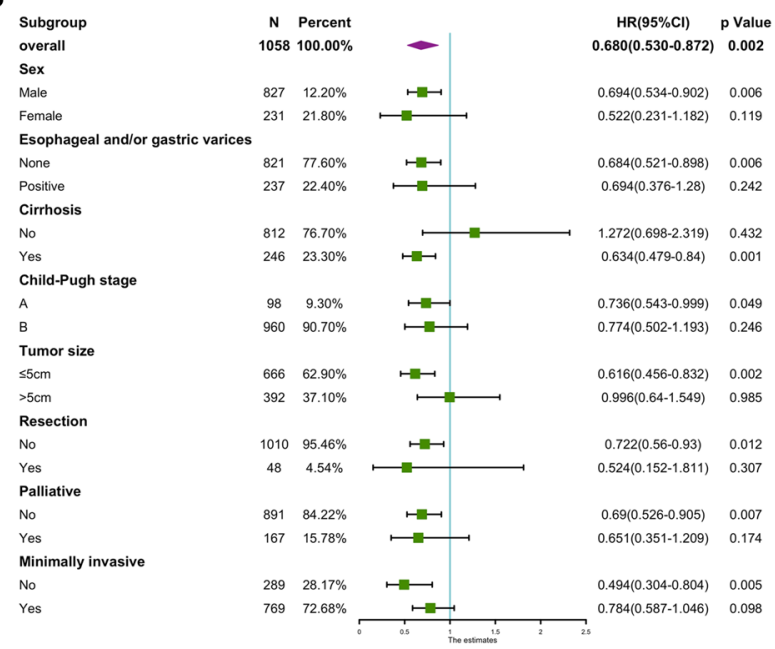

Fig. 4 Forest map comparing mortality risk in overall survival (A) and progression-free survival (B) between BCLC 0-B group HCC patients in $>45 y$ and age $\leq 45 y$ age groups. Numbers in parentheses are $95 \% \mathrm{Cls}$. $\mathrm{HR}$, hazard ratios; $\mathrm{Cl}$, confidence interval

in Asian men and 45 in women. Therefore, we evaluated $\mathrm{HCC}$ according to the BCLC staging in younger ( $\leq 45$ years) and older ( $>45$ years) patients.

Kim et al. [14] reported that younger patients had a worse prognosis compared to older patients, and Furuta et al. [15] found no significant difference in OS between younger patients and older patients with HCC. However, some studies showed that younger patients have better survival rates than older patients $[16,17]$ or better survival rates when treated with systemic chemotherapy [18]. This is consistent with the results of our study. In survival analysis based on the BCLC staging system, we showed that age affects the long-term survival of patients with BCLC stage $0-\mathrm{B}$ HCC. In BCLC stage $0-\mathrm{B}$, younger patients with $\mathrm{HCC}$ ( $\leq 45$ years) had better 5 -year OS and PFS compared to older patients with HCC ( $>45$ years).

Our data revealed a higher proportion of cirrhosis and worse Child-Pugh status in the older group, leading to better long-term survival in the younger group ( $\leq 45$ years) than in the older group ( $>45$ years). This may be because of the decrease in hepatic mass and hepatic blood flow with aging; additionally, the pharmacokinetics of many drugs change with age [19-21]. Zoli et al. [22] reported that portal venous blood flow rates were significantly lower in older patients than in younger patients. Younger patients have relatively better liver quality, whereas older patients show atrophy due to reduced liver cell counts. Thus, liver function is an important factor affecting prognosis, which is consistent with our findings [23]. Younger patients with HCC may have a higher likelihood of hepatic surrogate function, which is an important factor affecting prognosis [24]. Therefore, a better liver reserve in younger patients with HCC may help prolong survival after 5 years.

We analyzed the relationship between age, mortality, and different causes of death. We used restricted cubic sample bars to flexibly model and visualize the relationship between predicted age and all-cause mortality. In BCLC stage $0-B$, age was positively correlated with all-cause mortality and younger patients benefited, which is consistent with our previously observed results. There was a clear U-shaped relationship between age and all-cause mortality in BCLC C-D, with younger patients showing higher mortality rates and a more aggressive phenotype in late HCC compared to older patients [25]. Another study showed that despite having larger tumors and advanced tumor staging, younger patients tend to be treated more aggressively and frequently compared to patients aged 60 years or older, who tend to avoid aggressive treatment [14]. The relationship between the different causes of death and age was analyzed. According to a Japanese study, the risk rate of HCC-related or liverrelated death after hepatectomy was almost 1 , whereas the risk rate for other causes of death increased significantly with age [26]. However, Motoyama et al. [27] showed that although mortality from other causes is high in the elderly, the 5-year survival rate is lower in the elderly than in younger patients. This is consistent with our findings for the different causes of death in the two age groups of patients with HCC. At BCLC stage $0-B$, the cumulative incidence curves of $\mathrm{HCC}$ related deaths significantly differed $(p<0.05)$ (data not shown), with higher death rates in the older group 
than in the younger group, but the cumulative incidence curves of non-HCC-related deaths were essentially the same. Younger patients may be less likely than older patients to die from liver cancer at stage 0-B BCLC. The disease progresses differently in different stages in younger and older patients, which may help explain why previous studies showed differing results regarding whether age affects prognosis.

One study showed that younger patients typically present late with multiple lung metastases, whereas the disease is more likely to be detected in older patients during routine screening; therefore, the difference in reported clinicopathological features between the two groups may be related to differences in clinical presentation and screening strategies [5]. Another study also showed that young patients with HCC mostly had advanced disease at the time of detection and had larger tumors compared to the older group [15]. This suggests that early detection in younger patients, such as through screening, is beneficial, which is supported by our results.

This study had some limitations. First, this was a retrospective study and was somewhat constrained by differences in clinical characteristics between the two groups, resulting in selection bias. However, the homogeneity of the study population and combined data on risk factors minimized potential confounding factors. Second, this was a single-center study, and further research is needed to better understand the impact of age on the prognosis of liver cancer in other ethnicities or regions. Finally, our study focused on long-term survival outcomes, and additional studies are needed to evaluate short-term survival outcomes.

In conclusion, our study may be useful for predicting the prognosis of younger patients with HCC. In BCLC 0 -B stage, age affects the long-term prognosis of patients and is positively correlated with the mortality rate. Young patients with HCC with stage BCLC 0-B would benefit. Therefore, young patients with liver diseases, such as chronic hepatitis B, should undergo screening.

\section{Supplementary Information}

The online version contains supplementary material available at https://doi. org/10.1186/s12885-022-09293-x.

Additional file 1: Table S1. Baseline characteristics of study patients after propensity score analysis. Figure $\mathbf{S 1}$. The Kaplan-Meier survival curves of Overall survival (OS) and Progression-free survival (PFS) in BCLC 0-B group. (A-B) The OS (A) and PFS (B) in tumor size. (C-D) The OS (C) and PFS (D) in esophageal and/or gastric varices. (E-F) The OS (E) and PFS (F) Child staging $A$ and $B$.

\section{Acknowledgments}

The authors are grateful to the medical records department of the Beijing Ditan Hospital for their support.

\section{Authors' contributions}

ZYY, XHW and HWY designed the study; HWY drafted the manuscript and performed the statistical analysis; XHW participated in patient selection and implementation of the study; XLL and PW provided patient data; LHY and DDZ were responsible for the interpretation of data and revision. ZYY approved the final revision and approval of the manuscript. All authors read and approved the final manuscript.

\section{Funding}

This work was supported by the Special Fund of Capital Health Research and Development (No. 2020-2-2173), National Science Foundation of China (No. 81874435), Dengfeng Talent Support Program of Beijing Municipal Administration of Hospitals (No. DFL20191803), and Beijing Hospital Authority Clinical Medicine Development of Special Funding Support (code: ZYLX202127).

\section{Availability of data and materials}

The datasets used or analyzed during the current study are available from the corresponding author upon reasonable request.

\section{Declarations}

\section{Ethics approval and consent to participate}

The project was approved by the Ethics Committee of the Beijing Ditan Hospital, Capital Medical University, and was performed in accordance with the Declaration of Helsinki of 1975, as revised in 1983. Written informed consent was obtained from all patients. Information that could identify individual participants during or after data collection was not available.

\section{Consent for publication}

Not applicable.

\section{Competing interests}

The authors declare that they have no competing interests.

\section{Author details}

${ }^{1}$ Center of Integrative Medicine, Capital Medical University Affiliated Beijing Ditan Hospital, No. 8 Jing Shun East Street, Beijing 100015, People's Republic of China. ${ }^{2}$ Dongzhimen Hospital, Beijing University of Chinese Medicine,

Chaoyang District, Beijing 100029, People's Republic of China.

Received: 30 January 2021 Accepted: 9 February 2022

Published online: 18 February 2022

\section{References}

1. Arzumanyan A, Reis HMGPV, Feitelson MA. Pathogenic mechanisms in HBV- and HCV-associated hepatocellular carcinoma. Nat Rev Cancer. 2013;13(2):123-35.

2. Bosch FX, Ribes J, Díaz M, Cléries R. Primary liver cancer: worldwide incidence and trends. Gastroenterology. 2004;127(5 Suppl 1):S5-S16.

3. Finianos A, Matar CF, Taher A. Hepatocellular Carcinoma in $\beta$-Thalassemia Patients: Review of the Literature with Molecular Insight into Liver Carcinogenesis. Int J Mol Sci. 2018;19(12):4070.

4. Johnson PJ, Berhane S, Kagebayashi C, Satomura S, Teng M, Reeves $\mathrm{HL}$, et al. Assessment of liver function in patients with hepatocellular carcinoma: a new evidence-based approach-the ALBI grade. J Clin Oncol. 2015;33(6):550-8.

5. Lam CM, Chan AOO, Ho P, Ng IOL, Lo CM, Liu CL, et al. Different presentation of hepatitis B-related hepatocellular carcinoma in a cohort of 1863 young and old patients - implications for screening. Aliment Pharmacol Ther. 2004;19(7):771-7.

6. Yamazaki Y, Kakizaki S, Sohara N, Sato K, Takagi H, Arai H, et al. Hepatocellular carcinoma in young adults: the clinical characteristics, prognosis, and findings of a patient survival analysis. Dig Dis Sci. 2007;52(4):1 103-7.

7. Chang $\mathrm{P}-\mathrm{E}$, Ong $\mathrm{W}-\mathrm{C}$, Lui $\mathrm{H}-\mathrm{F}$, Tan C-K. Is the prognosis of young patients with hepatocellular carcinoma poorer than the prognosis of older patients? A comparative analysis of clinical characteristics, prognostic features, and survival outcome. J Gastroenterol. 2008;43(11):881-8. 
8. Kaibori M, Matsui K, Ishizaki M, Saito T, Kitade H, Matsui Y, et al. Hepatic resection for hepatocellular carcinoma in the elderly. J Surg Oncol. 2009;99(3):154-60.

9. Yamamoto K, Takenaka K, Matsumata T, Shimada M, Itasaka H, Shirabe $\mathrm{K}$, et al. Right hepatic lobectomy in elderly patients with hepatocellular carcinoma. Hepatogastroenterology. 1997;44(14):514-8.

10. Grieco A, Pompili M, Caminiti G, Miele L, Covino M, Alfei B, et al. Prognostic factors for survival in patients with early-intermediate hepatocellular carcinoma undergoing non-surgical therapy: comparison of Okuda, CLIP, and BCLC staging systems in a single Italian Centre. Gut. 2005;54(3):411-8.

11. EASL-EORTC clinical practice guidelines. Management of hepatocellular carcinoma. J Hepatol. 2012;56(4):908-43.

12. Heimbach JK, Kulik LM, Finn RS, Sirlin CB, Abecassis MM, Roberts LR, et al. AASLD guidelines for the treatment of hepatocellular carcinoma. Hepatology (Baltimore, Md). 2018;67(1):358-80.

13. Lee CR, Lim JH, Kim SH, Ahn SH, Park YN, Choi GH, et al. A comparative analysis of hepatocellular carcinoma after hepatic resection in young versus elderly patients. J Gastrointest Surg. 2012;16(9):1736-43.

14. Kim JH, Choi MS, Lee H, Kim DY, Lee JH, Koh KC, et al. Clinical features and prognosis of hepatocellular carcinoma in young patients from a hepatitis B-endemic area. J Gastroenterol Hepatol. 2006;21 (3):588-94.

15. Furuta T, Kanematsu T, Matsumata T, Shirabe K, Yamagata M, Utsunomiya T, et al. Clinicopathologic features of hepatocellular carcinoma in young patients. Cancer. 1990;66(11):2395-8.

16. Kim JM, Cho BI, Kwon CHD, Joh J-W, Park JB, Lee JH, et al. Hepatectomy is a reasonable option for older patients with hepatocellular carcinoma. Am J Surg. 2015;209(2):391-7.

17. Lee J-H, Cho Y, Kim HY, Cho EJ, Lee DH, Yu SJ, et al. Serum tumor markers provide refined prognostication in selecting liver transplantation candidate for hepatocellular carcinoma patients beyond the Milan criteria. Ann Surg. 2016;263(5):842-50.

18. Poulou LS, Botsa E, Thanou I, Ziakas PD, Thanos L. Percutaneous microwave ablation vs radiofrequency ablation in the treatment of hepatocellular carcinoma. World J Hepatol. 2015;7(8):1054-63.

19. Davies EA, O'Mahony MS. Adverse drug reactions in special populations the elderly. Br J Clin Pharmacol. 2015;80(4):796-807.

20. Saleh DO, Mansour DF, Hashad IM, Bakeer RM. Effects of sulforaphane on D-galactose-induced liver aging in rats: role of keap-1/nrf-2 pathway. Eur J Pharmacol. 2019;855:40-9.

21. Stader F, Siccardi M, Battegay M, Kinvig H, Penny MA, Marzolini C. Repository describing an aging population to inform physiologically based pharmacokinetic models considering anatomical, physiological, and biological age-dependent changes. Clin Pharmacokinet. 2019;58(4):483-501.

22. Zoli M, lervese T, Abbati S, Bianchi GP, Marchesini G, Pisi E. Portal blood velocity and flow in aging man. Gerontology. 1989;35(2-3):61-5.

23. Carr BI, Guerra V. Hepatocellular carcinoma Extrahepatic metastasis in relation to tumor size and alkaline phosphatase levels. Oncology. 2016;90(3):136-42.

24. Llovet JM, Montal R, Sia D, Finn RS. Molecular therapies and precision medicine for hepatocellular carcinoma. Nat Rev Clin Oncol. 2018;15(10):599-616.

25. Heo MJ, Kim YM, Koo JH, Yang YM, An J, Lee S-K, et al. microRNA-148a dysregulation discriminates poor prognosis of hepatocellular carcinoma in association with USP4 overexpression. Oncotarget. 2014:5(9):2792-806.

26. Kaibori M, Yoshii K, Yokota I, Hasegawa K, Nagashima F, Kubo S, et al. Impact of advanced age on survival in patients undergoing resection of hepatocellular carcinoma: report of a Japanese Nationwide survey. Ann Surg. 2019:269(4):692-9.

27. Motoyama H, Kobayashi A, Yokoyama T, Shimizu A, Sakai H, Furusawa N, et al. Impact of advanced age on the short- and long-term outcomes in patients undergoing hepatectomy for hepatocellular carcinoma: a singlecenter analysis over a 20-year period. Am J Surg. 2015;209(4):733-41.

\section{Publisher's Note}

Springer Nature remains neutral with regard to jurisdictional claims in published maps and institutional affiliations.

Ready to submit your research? Choose BMC and benefit from:

- fast, convenient online submission

- thorough peer review by experienced researchers in your field

- rapid publication on acceptance

- support for research data, including large and complex data types

- gold Open Access which fosters wider collaboration and increased citations

- maximum visibility for your research: over $100 \mathrm{M}$ website views per year

At BMC, research is always in progress.

Learn more biomedcentral.com/submissions 